Relations industrielles

Industrial Relations

\title{
Professional Workers and Collective Bargaining, by Shirley B. Goldenberg, Study no 2, Task Force on Labour Relations, Ottawa, Privy Council Office, 1968, 298 pp.
}

\section{André Roy}

Volume 25, numéro 3, 1970

URI : https://id.erudit.org/iderudit/028169ar

DOI : https://doi.org/10.7202/028169ar

Aller au sommaire du numéro

\section{Éditeur(s)}

Département des relations industrielles de l'Université Laval

\section{ISSN}

0034-379X (imprimé)

1703-8138 (numérique)

Découvrir la revue

Citer ce compte rendu

Roy, A. (1970). Compte rendu de [Professional Workers and Collective Bargaining, by Shirley B. Goldenberg, Study no 2, Task Force on Labour Relations, Ottawa, Privy Council Office, 1968, 298 pp.] Relations industrielles / Industrial Relations, 25(3), 627-628. https://doi.org/10.7202/028169ar

Tous droits réservés @ Département des relations industrielles de l'Université Laval, 1970
Ce document est protégé par la loi sur le droit d'auteur. L’utilisation des services d’Érudit (y compris la reproduction) est assujettie à sa politique d'utilisation que vous pouvez consulter en ligne.

https://apropos.erudit.org/fr/usagers/politique-dutilisation/ 
Professional Workers and Collective Bargaining, by Shirley B. Goldenberg, Study no 2, Task Force on Labour Relations, Ottawa, Privy Council Office, 1968, 298 pp.

Depuis une dizaine d'années, la transformation de la vie sociale et économique a modifié considérablement les structures des professions. Le phénomène est le suivant : d'une part, on compte un nombre de plus en plus grand de membres des professions libérales traditionnelles qui sont devenus des salariés; d'autre part, plusieurs professions nouvelles ont pris naissance par suite des découvertes scientifiques et de leurs applications et certains groupes déjà existants ont accédé graduellement au statut professionnel, et la grande majorité d'entre eux sont aussi des salariés.

Les chiffres, d'ailleurs, parlent éloquemment. En 1931, il y avait au Canada quelque 238,00 professionnels de toutes catégories; en 1961, leur nombre était passé à 634,000 , soit une augmentation de 166 pour cent. Et, depuis dix ans, on peut penser que cette tendance s'est encore accentuée.

Mais ce qui est encore plus important, la proportion du nombre des professionnels salariés a augmenté davantage. Si l'on fait exception des dentistes, des avocats et des notaires, dans chacun des secteurs professionnels, le nombre des salariés dépassait le tiers et, dans quelques cas, on peut affirmer que toute la profession globalement était salariée.

Ces faits ne pouvaient pas ne pas soulever le problème des rapports collectifs de travail pour les salariés professionnels. Cette question a fait le sujet d'une étude particulière fort intéressante et très pratique aussi dans l'entreprise considérable qu'a été l'Equipe spécialisée en relations du travail. C'est l'étude numéro 2 dont l'auteur est Shirley B. Goldenberg.

Dans ce travail de 280 pages environ, qui contient en outre un résumé substantiel en français, elle étudie d'abord le problème de la négociation collective chez les salariés professionnels tel qu'il se présente à l'heure actuelle au Canada. Après avoir consacré un chapitre aux différents facteurs qui sont de nature à exercer une influence sur l'implantation des régimes de la convention collective parmi ces catégories de salariés dont l'identification n'est pas toujours facile, l'auteur considère tour à tour les réactions des professionnels eux-mêmes en présence de cette situation nouvelle, la situation juridique des salariés professionnels face à la législation générale du travail, l'établissement des mécanismes de négociation appropriés, les méthodes et l'objet des négociations et, finalement, les implications de ce phénomène nouveau sur la vie publique.

Pour étayer son travail, Shirley Goldenberg a scruté le cas particulier des ingénieurs qui avait fait plus tôt l'objet d'un rapport déposé à l'Equipe spécialisée.

Il est difficile, en quelques paragraphes, d'analyser ce travail considérable. Ce qu'on peut en retenir, c'est que nous nous trouvons en présence d'un phénomène d'une ampleur considérable et qui ne cessera de s'accentuer dans l'avenir.

On ne peut ici que dégager quelquesunes des idées générales qui inciteront tous ceux qui s'intéressent aux rapports collectifs de travail à lire et approfondir cette étude. 1 - A l'intérieur même des professions, si l'on fait exception des infirmières et des enseignants, il y a réticence de la part des corps professionnels eux-mêmes à accepter l'idée de la négociation collective. 2 - Tous les groupes professionnels tiennent fort, indépendamment de leur importance numérique dans l'ensemble de la main d'oeuvre salariée, à conserver leur propre identité, à avoir leur propre unité de négociation. 3 - Les membres de certaines catégories professionnelles, en particulier les médecins, à cause de l'établissement des régimes d'assurancesanté, sans être véritablement des salariés, se trouvent placés dans une situation où il leur faut maintenant négocier à tout prix les barêmes de leurs honoraires. 4 - L'insertion dans les conventions collectives de dispositions relatives aux standards professionnels constitue une question difficile et controversée. 5 - La réglementation du droit de grève pose des problèmes particuliers là où la santé et la sécurité des citoyens sont en jeu. 6 - Les membres de la plupart des professions s'opposent en règle générale à faire partie des centrales syndicales existantes. 7 - Il ressort de l'étude que, à part la Saskatchewan, la province de Québec est celle qui, lors de l'étude 
du Code du travail et de la Loi de la fonction publique, a fait le plus d'effort en vue de trouver une solution aux problèmes des rapports collectifs des salariés professionnels, quoique, depuis, le gouvernement central ait emboîté le pas et que, dans d'autres provinces, des commissions d'enquête aient étudié la question ou des projets de lois aient été ébauchés.

Ces quelques faits ne donnent qu'une pâle image de la valeur du travail de Mme Goldenberg qui, non seulement constitue un instrument essentiel pour ceux qui ont à appliquer la législation du travail telle qu'elle existe présentement aux diverses catégories professionnelles ou à mettre au point des lois et des réglementations nouvelles, mais est de nature à aider tous ceux qui s'intéressent aux problèmes du travail à se former une opinion sérieuse sur un phénomène nouveau de la vie sociale.

\section{André ROY}

Pourquoi et comment déléguer, par Roland Claude, Paris, Entreprise Moderne d'Edition, Collection Cadréco, no 10, 1969, 142 pp.

On reçonnaît facilement qu'uncun système d'organisation n'est viable et qu'aucune activité commune n'est possible sans une délégation de l'autorité. C'est tellement vrai qu'on peut attribuer la disparition de quelques petites et moyennes entreprises québécoises à l'incapacité du chef ou du propriétaire de déléguer un peu de son autorité à ses collaborateurs immédiats. Savoir déléguer judicieusement son autorité est une qualité qu'un administrateur peut acquérir ou améliorer. Dans cette optique, une lecture rapide de ce petit volume de la Collection Cadreco peut être grandement utile. Il tente de répondre aux questions fondamentales telles que: « Comment déléguer? - Comment choisir la personne à qui on veut déléguer son autorité ? - Quels sont les types d'autorité qui peuvent être délégués? Quelle est la latitude qu'on peut accorder à celui à qui on délègue son autorité ?

$\mathrm{Au}$ premier abord, le volume nous apparaît comme un recueil de recettes, mais cette impression se dissipe rapidement, lorsqu'on s'arrête aux idées principales clairement exprimées qui sont autant de réflexions profondes sur chacune des questions soulevées. C'est aussi un recueil et une mise en ordre intelligente de l'information éparse qu'on retrouve sur le sujet dans les différents ouvrages d'administration.

\section{Laurent BELANGER}

\section{Comment rédiger vos rapports, par Geor-} ges Bousquie, Paris, Entreprise Moderne d'Edition, Collection Cadréco, no 11 , (8e édition - 50e mille) 1970 , $150 \mathrm{pp}$.

A l'heure où la qualité de la communication dans l'entreprise moderne prend une importance telle qu'elle a des répercussions sur tous les aspects de ses activités, aussi bien sur la production que sur la mise en marché en passant par les relations du travail, ce petit livre vient apporter de judicieux conseils sur la façon de rédiger divers types de rapports.

Sa valeur tient surtout au fait qu'il est le fruit de l'expérience.

D'ailleurs de nombreux exemples de rapports aussi variés que concis viennent illustrer la façon dont on peut communiquer des renseignements relatifs à divers aspects de la vie de l'entreprise tels que: bris de machine, accident du travail, présentation d'un nouveau produit, etc.

\section{Jean BERNIER}

Le perfectionnement des cadres dirigeants et les politiques et pratiques en matière d'administration du personnel en Asie, Relations professionnelles, \# 35, Genève, B.I.T., 1969, 141 pp.

Le présent numéro de la série Relations professionnelles nous est présenté comme le compte rendu des travaux de la Commission du perfectionnement des cadres dirigeants à l'occasion de la sixième Conférence régionale asienne de I'O.I.T., tenue à Tokyo du 2 au 14 septembre 1968.

L'ouvrage compte deux parties. D'abord le rapport sur \& le perfectionnement des cadres dirigeants $\gg$ préparé par le BIT pour servir de base à la 